\title{
Surface phase transitions in free-standing films of nonchiral tilted hexatic liquid crystals
}

\author{
Chih-Yu Chao, ${ }^{1, *}$ Tung-Cheng Pan, ${ }^{1}$ and John T. $\mathrm{Ho}^{2}$ \\ ${ }^{1}$ Department of Physics, National Taiwan University, Taipei 10617, Taiwan, Republic of China \\ ${ }^{2}$ Department of Physics, University at Buffalo, The State University of New York, Buffalo, New York 14260
}

(Received 9 January 2003; published 14 April 2003)

\begin{abstract}
Surface freezing transitions in free-standing films of achiral Schiff's-base liquid crystals 50.6 and 70.7 exhibiting tilted hexatic phases have been studied using optical textures. The evolution of textures with temperature in 50.6 films is qualitatively similar to that reported earlier in the ferphenyl ester FTE1, suggesting the existence of the surface smectic- $L$ phase, except that the stripe texture in 50.6 consists of alternating light and dark stripes of unequal widths. No stripe texture is observed in 70.7.
\end{abstract}

DOI: 10.1103/PhysRevE.67.040702

Smectic liquid-crystal (LC) systems have a rich variety of phases with different types of in-plane two-dimensional order. Among the most interesting are the tilted hexatic phases, which can differ from each other in the relation between the in-plane bond directions and the tilt direction. In the smectic$I(\mathrm{Sm}-I)$ phase, the tilt direction is along one of the local bonds, while in the smectic- $F(\mathrm{Sm}-F)$ phase, it is halfway between two local bonds. A direct $\mathrm{Sm}-I-\mathrm{Sm}-F$ transition is typically observed experimentally $[1,2]$. Earlier studies on a lyotropic LC suggested that a new smectic- $L(\mathrm{Sm}-L)$ phase, in which the tilt direction lies along an intermediate angle between $0^{\circ}$ and $30^{\circ}$ from a local bond, may also exist [3-5], although it was unclear whether the phase is hexatic or multicrystalline. Theoretically, it has been shown that an intermediate Sm- $L$ phase can indeed occur between the Sm- $I$ and $\mathrm{Sm}-F$ phases $[6,7]$. The possible existence of the hexatic Sm$L$ phase in a thermotropic LC was first found in optical studies of free-standing films of the terphenyl ester FTE1, where the occurrence of a modulated stripe texture was interpreted as an indicative of a surface $\mathrm{Sm}-L$ phase [8,9]. This structural interpretation was subsequently confirmed in FTE1 films by electron diffraction (ED) $[10,11]$.

We report here the results of optical studies of the textures in free-standing films of the Schiff's-base compounds 50.6 (4- $n$-hexyl- $N$-[4- $n$-pentyloxy-benzylidene]-aniline) and 70.7 (4-n-heptyloxybenzylidene-4- $n$-heptylaniline). Earlier optical studies have reported stripe textures indicative of a $\mathrm{Sm}-L$ phase on the surface of a smectic- $C(\mathrm{Sm}-C)$ interior in both 50.6 and 70.7, similar to that in FTE1 [8,9]. However, subsequent experiments on these materials have raised questions about the nature of their surface-freezing behavior. First, in 50.6, a more recent optical study has reported unusual sequences of textures and phases, some in the presence of the smectic- $A(\mathrm{Sm}-A)[12]$, which may not be totally consistent with the earlier observations [8,9]. Second, in 70.7, recent detailed ED studies on the structure of free-standing films $[13,14]$ have shown no evidence for the existence of a surface $S \mathrm{Sm}-L$ phase that was suggested in the optical experiments $[8,9]$. Our current optical studies are aimed at clarifying these conflicting results.

Our Schiff's-base compounds 50.6 and 70.7 were recrys-

\footnotetext{
*Author to whom correspondence should be addressed. Email address: cychao@phys.ntu.edu.tw
}

PACS number(s): 61.30.Eb, 64.70.Md

tallized to achieve high chemical purity. Free-standing films of these materials were drawn in the $\mathrm{Sm}-A$ or $\mathrm{Sm}-C$ phase over a hole of $3 \times 10 \mathrm{~mm}^{2}$ in a glass cover slip in a hot stage whose temperature was controlled with a resolution of $0.01{ }^{\circ} \mathrm{C}$, with the film spreader beneath the supporting glass slip. Images were recorded using a polarizing microscope in the reflection mode. Due to the chemical instability of the hydrolysis-prone Schiff's-base compounds, the hot stage in our studies was specially designed to be in a closed system that was evacuated and backfilled with 0.5 atm of pure argon gas to minimize sample hydrolysis, a precaution that was not taken in earlier optical experiments. We have made detailed studies of the textures of films from 4 to 20 molecular layers in 50.6 and 8 to 20 layers in 70.7 while cooling through the $\mathrm{Sm}-\mathrm{C}$-hexatic transition. No significant drifts in their phase transition temperatures were observed in our measurements.

We first describe the results in 50.6. In general, the phase transition temperatures decrease with increasing film thickness. The temperatures reported below are approximate values applicable to the thicker films of 15-20 molecular layers. The texture of the films in the $\mathrm{Sm}-\mathrm{C}$ phase above $58^{\circ} \mathrm{C}$ is characterized by rapid fluctuations in the c-director field. Broad, fluctuating extinction brushes emanating from point defects represent typical features of the optical texture of this phase, as shown in Fig. 1. Upon cooling to just below $58^{\circ} \mathrm{C}$, the c-director fluctuations are largely reduced, leaving a large-scale, frozen texture of fairly uniform domains separated by walls. The walls are similar to that observed in Refs. $[8,9]$. At slightly lower temperatures, the films display an array of thin disclination lines spaced $0.5-1 \mu \mathrm{m}$ apart. The

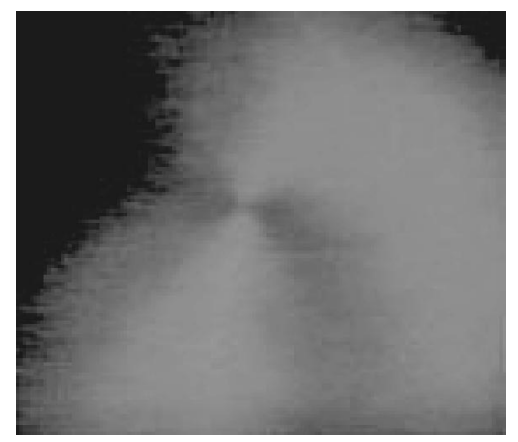

FIG. 1. Broad, fluctuating extinction Sm-C brushes emanating from point defect in a 50.6 film at $60^{\circ} \mathrm{C}$. The horizontal dimension is about $250 \mu \mathrm{m}$. 


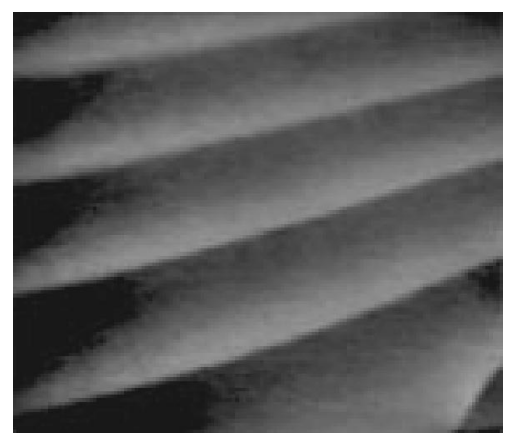

FIG. 2. Line texture (splay texture) in a 50.6 film at $50.8^{\circ} \mathrm{C}$ showing typical modulated sharp lines separating bands with continuously changing intensity. The horizontal dimension is about $150 \mu \mathrm{m}$.

director orientation between each pair of lines is initially difficult to resolve but on lowering the temperature the lines move further apart and each band is seen to have an obvious gradation from dark to bright, implying that the director rotates continuously across each band. This line texture is thus composed of bands of continuous director splay, separated by splay disclinations, which is typically observed in the Sm$I$ or Sm- $F$ phase. Meanwhile, some faint c-director fluctuations are still observable. Since a similar line texture was observed in FTE1 $[8,9]$ in a temperature range where ED study has indicated the coexistence of Sm- $I$ surfaces and a Sm- $C$ interior [10], we suggest that the structure in 50.6 at this temperature range is also similar. This texture is found to reappear with more clarity in 50.6 below $51^{\circ} \mathrm{C}$, which is shown in Fig. 2.

In 50.6 films below $55^{\circ} \mathrm{C}$, this line texture transforms into that of parallel, uniform stripes of alternating brightness and darkness. The stripes are found to be parallel to the original line texture, each splay band typically evolving into precisely one light and one dark stripe with unequal widths, as shown in Fig. 3. Upon cooling, the period of both types of stripes increases with decreasing temperature with the width of the narrower stripe increasing at a faster rate than that of the wider stripe as the temperature is decreased. Between crossed polarizers, the alternating bright and dark stripes denote variations of the azimuthal orientations of the c-director.

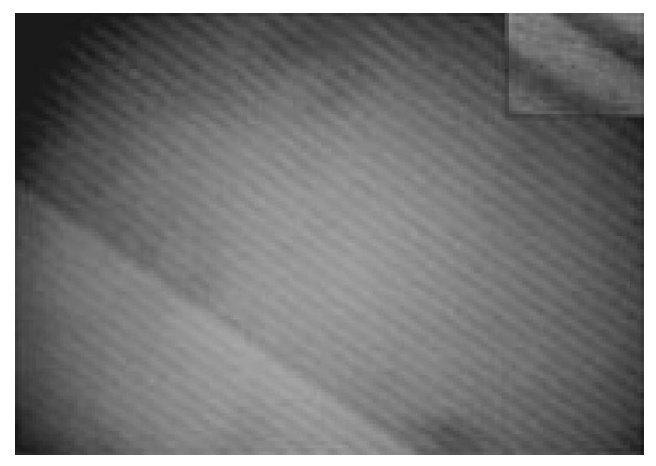

FIG. 3. Stripe texture in a 50.6 film at $54^{\circ} \mathrm{C}$, showing alternating light and dark uniform bands of unequal widths. The horizontal dimension is about $200 \mu \mathrm{m}$. The inset shows details of the adjacent light and dark stripes.
By slowly rotating the microscope hot stage, one may observe that the stripes become darkest when the c-director is either parallel or perpendicular to the polarizers, whereas the stripes become brightest when the c-director makes a $45^{\circ}$ angle with the polarizers. The director orientation appears to be uniform within each stripe and jumps by about $30^{\circ}$ when crossing a stripe boundary, which is a sharp, weakly undulating wall. The stripe texture observed here is qualitatively similar to that in Refs. [8,9], with the noted exception that the widths of the alternating light and dark stripes are equal in FTE1 but unequal in 5O.6. It should also be noted that the typical stripe width in 50.6 is about $1 / 4$ that in FTE1. Interior c-director fluctuations are still observable in the background throughout the stripe phase in 50.6, although they become less visible with decreasing temperature. Since the stripe texture in FTE1 has been identified as indicative of $\mathrm{Sm}-L$ surfaces coexisting with a $\mathrm{Sm}-C$ interior, we speculate that the structure of the 50.6 films in this temperature range may also be similar. Additional ED studies will be needed to confirm this. At this point, the origin of the unequal widths of the alternating stripes is unclear. We can speculate on the possible role of splay chirality [15] or tilt modulations [16] that have led to qualitatively similar textures in other phases.

Below $53{ }^{\circ} \mathrm{C}$, the widths of both the light and dark stripes increase with decreasing temperature, but at different rates, until they eventually become equal. Subsequently, a brick wall pattern is observed, as shown in Fig. 4(a). Through relative elongation and contraction of successive bend wall segments, the brick wall pattern gradually transforms into an elongated hexagonal texture, and then into a honeycomb texture on cooling, as shown in Fig. 4(b). At $51^{\circ} \mathrm{C}$, the director fluctuations and domain wall undulations are quenched and the stripes slowly lose their modulated texture and gradually expand to much broader splay bands, producing a texture similar to that seen between $58{ }^{\circ} \mathrm{C}$ and $55^{\circ} \mathrm{C}$, as shown in Fig. 2. Based on a similar texture observed in FTE1 $[8,9]$ and its structural identification using ED [10], we suggest that the 50.6 films are in the $\mathrm{Sm}-F$ phase below $51^{\circ} \mathrm{C}$. Our observations in 50.6 are summarized in the phase diagram in Fig. 5. It should be noted that 50.6 films less than six layers thick do not exhibit any stripe texture throughout the hexatic range, but instead retain the coarse texture of large-scale correlated regions frozen in from the $\mathrm{Sm}-C$ phase. We speculate that, in very thin films, as the two surfaces start to interact and the c-director is constrained to be uniform through the film, the $S m-L$ is no longer a chiral phase and stripe domain formation would be inhibited.

In 70.7 films above $77.7^{\circ} \mathrm{C}$, the texture shows rapid fluctuations in the c-director field, consistent with earlier ED results showing $\mathrm{Sm}-C$ surfaces coexisting with a $\mathrm{Sm}-A$ interior [13]. At $77.7^{\circ} \mathrm{C}$, the director fluctuations are quenched, leaving a frozen texture of fairly uniform splay domains separated by thin disclination lines. This splay texture, which persists down to $69^{\circ} \mathrm{C}$, is similar to that in Fig. 2. It is composed of bands of continuous director splay, which is characteristic of the Sm-I phase [2], and is again consistent with ED observations [13]. More importantly, we observe no stripe texture between $77.7^{\circ} \mathrm{C}$ and $69^{\circ} \mathrm{C}$ in 70.7 films, in contrast to earlier optical studies $[8,9]$. In this temperature 

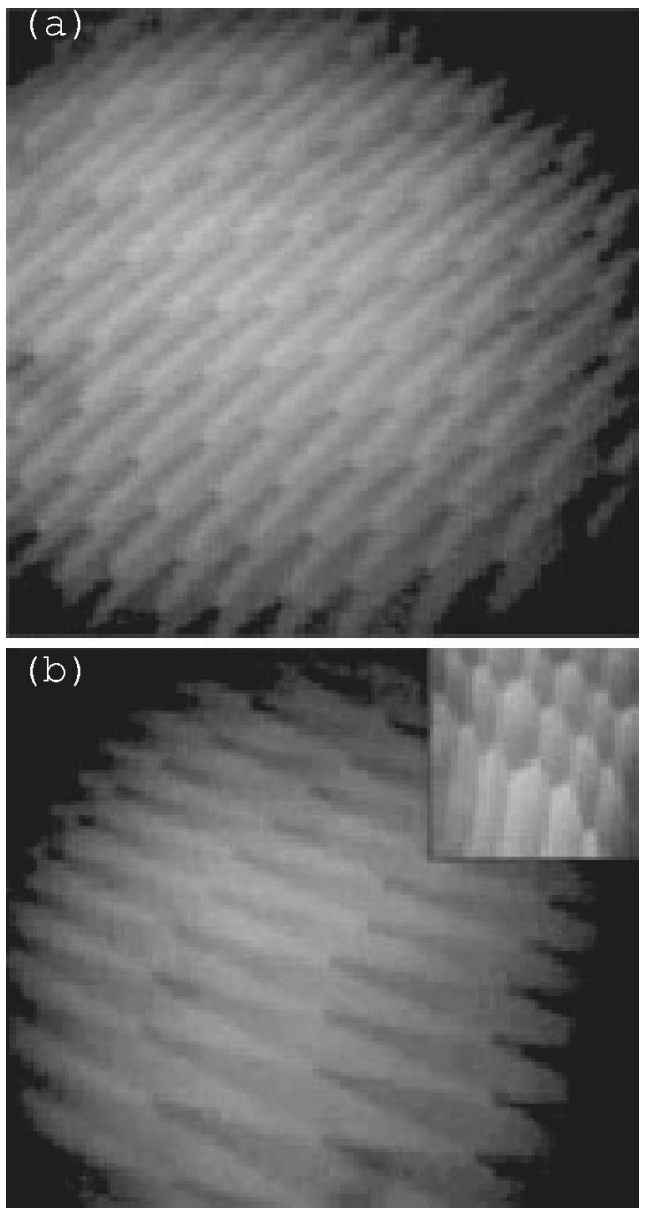

FIG. 4. (a) Brick wall texture in a 50.6 film at $52^{\circ} \mathrm{C}$. (b) Elongated hexagonal texture at about $51.8^{\circ} \mathrm{C}$. The inset shows a honeycomb texture at about $51.5^{\circ} \mathrm{C}$. The horizontal dimensions are about $250 \mu \mathrm{m}$ for (a), $200 \mu \mathrm{m}$ for (b), and $100 \mu \mathrm{m}$ for the inset in (b).

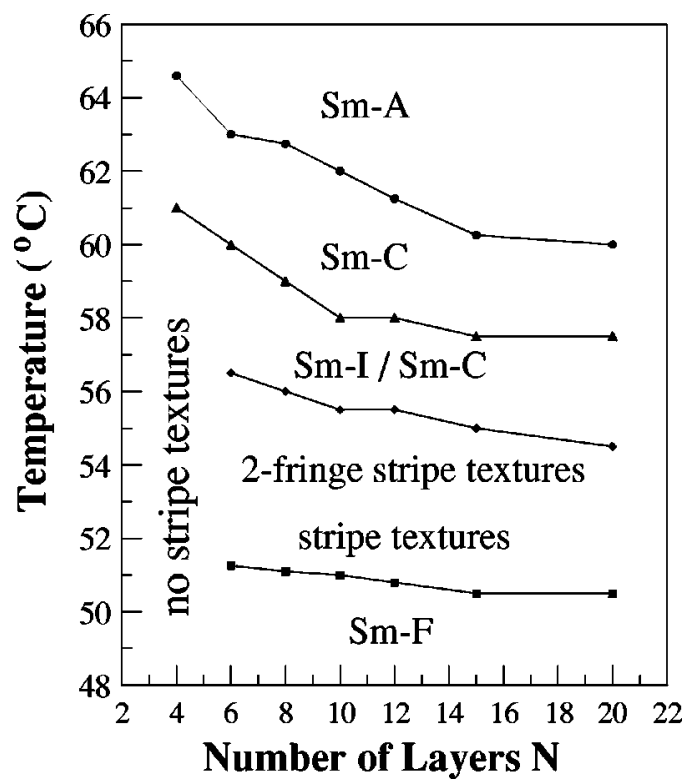

FIG. 5. Optical phase diagram for 50.6.

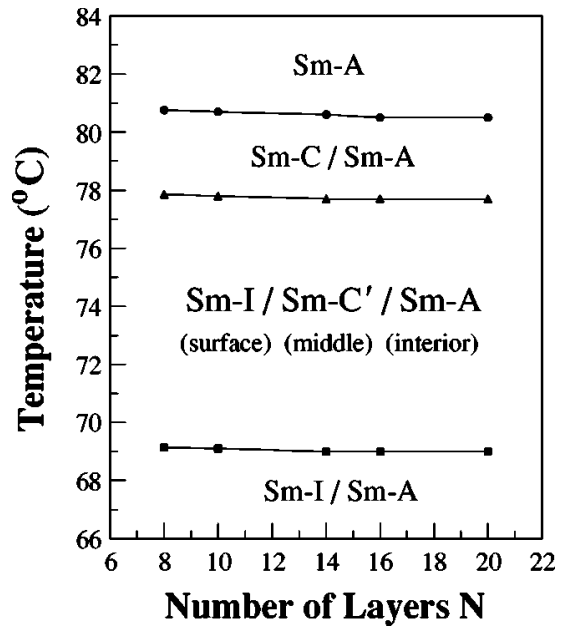

FIG. 6. Phase diagram for 70.7 with $\mathrm{Sm}-I / \mathrm{Sm}-C^{\prime} / \mathrm{Sm}-A$ denoting the coexistence, as a function of distance from the surface, of three distinct phases: an outermost Sm- $I$ layer, several layers of a middle Sm- $C^{\prime}$ phase, and a Sm- $A$ interior [13].

range, we also do not observe c-director fluctuations, which is consistent with our previous ED results indicating that the interior layers are in the $\mathrm{Sm}-C^{\prime}$ and $\mathrm{Sm}-A$ phases [13], but not the $\mathrm{Sm}-C$ phase, as suggested by earlier x-ray experiments [17]. The Sm- $C^{\prime}$ phase is believed to be a tilted phase with hexaticlike positional correlations but no long-range bond-orientational order [13]. Since the $\mathrm{Sm}-C^{\prime}$ phase is more strongly correlated than the $\mathrm{Sm}-C$ phase, it is reasonable to expect c-director fluctuations to be suppressed. Upon further cooling, the middle $\mathrm{Sm}-\mathrm{C}^{\prime}$ phase transforms to the $\mathrm{Sm}-I$ phase at $69^{\circ} \mathrm{C}$ [14]. The phase diagram for 70.7 films is summarized in Fig. 6.

Our results in 50.6 do not agree in significant ways with those in Ref. [12], that described sequences of phases and textures that are quite different from ours. However, in view of the fact that Ref. [12] mentioned no special effort to prevent sample hydrolysis and reported transition temperatures that are typically $8^{\circ} \mathrm{C}$ lower than ours, we suspect that sample degradation may be the cause of the discrepancy.

In summary, our optical results in 70.7 films are consistent with earlier ED structural data on the surface freezing of these films [13]. However, our results differ from earlier optical studies $[8,9]$ as we do not observe any stripe texture in 70.7 films. Our results in 50.6 films are qualitatively similar to those seen earlier in FTE1 $[8,9]$ and suggest that the surface freezing behavior of 50.6 may also involve the $\mathrm{Sm}-L$ phase just as in FTE1 [10]. Additional ED studies on 50.6 films to confirm the structure are warranted. Unexpectedly and perhaps significantly, the stripe texture we observe in 50.6 films consists of alternating light and dark stripes of unequal widths, which evolve to be equal at lower temperatures.

We are grateful to B. N. Chang and P. J. Wu for participation in the early phase of this work, to J. E. Maclennan for helpful discussions, and to W. B. Shu for technical assistance. This work was supported by the National Science Council, Taiwan, Republic of China and Chunghwa Picture Tube Company. 
[1] S.B. Dierker, R. Pindak, and R.B. Meyer, Phys. Rev. Lett. 56, 1819 (1986).

[2] E.B. Sirota, P.S. Pershan, L.B. Sorensen, and J. Collett, Phys. Rev. A 36, 2890 (1987).

[3] G.S. Smith, E.B. Sirota, C.R. Safinya, and N.A. Clark, Phys. Rev. Lett. 60, 813 (1988).

[4] E.B. Sirota, G.S. Smith, C.R. Safinya, R.J. Plano, and N.A. Clark, Science 242, 1406 (1988).

[5] G.S. Smith, E.B. Sirota, C.R. Safinya, R.J. Plano, and N.A. Clark, J. Chem. Phys. 92, 4519 (1990).

[6] J.V. Selinger and D.R. Nelson, Phys. Rev. Lett. 61, 416 (1988).

[7] J.V. Selinger and D.R. Nelson, Phys. Rev. A 39, 3135 (1989).

[8] J.E. Maclennan and M. Seul, Phys. Rev. Lett. 69, 2082 (1992).

[9] J.E. Maclennan, U. Sohling, N.A. Clark, and M. Seul, Phys. Rev. E 49, 3207 (1994).
[10] C.Y. Chao, S.W. Hui, J.E. Maclennan, C.F. Chou, and J.T. Ho, Phys. Rev. Lett. 78, 2581 (1997).

[11] C.Y. Chao, J.E. Maclennan, J.Z. Pang, S.W. Hui, and J.T. Ho, Phys. Rev. E 57, 6757 (1998).

[12] E.I. Demikhov and V.K. Dolganov, JETP Lett. 64, 32 (1996).

[13] C.Y. Chao, S.W. Hui, and J.T. Ho, Phys. Rev. Lett. 78, 4962 (1997).

[14] C.Y. Chao, C.R. Lo, and J.T. Ho, Phys. Rev. E 61, 5407 (2000).

[15] R. Najjar and Y. Galerne, Mol. Cryst. Liq. Cryst. 367, 395 (2001).

[16] Y. Yabe, N. Shen, E. Mazur, and H. Yokoyama, Phys. Rev. Lett. 82, 759 (1999).

[17] E.B. Sirota, P.S. Pershan, S. Amador, and L.B. Sorensen, Phys. Rev. A 35, 2283 (1987). 